\section{Military Technical College Kobry El-Kobbah, Cairo, Egypt}



\author{
$7^{\text {th }}$ International Conference \\ on Electrical Engineering \\ ICEENG 2010
}

\title{
Orbit Determination Using Spread Spectrum Ranging
}

\author{
By \\ M. I. Youssef* \\ M. Zahara* \\ A. E. Emam* \\ M. Abd ElGhany*
}

\section{$\underline{\text { Abstract: }}$}

In this paper, analysis on precise geostationary satellite orbit determination using spread spectrum ranging measurements is performed. Adding to the communication system a residue number system block to enhance its performance. The results are compared to that provided from orbit determination using both dual RF-tone ranging from two separate earth station locations and classical orbit determination using both angular measurement and RF-tone measurements from an earth station.

A model for the spread spectrum ranging system is developed to be used in the orbit determination process. Chaotic code generator is used instead of conventional PN code generators in the spread spectrum ranging system. The performance of the system is evaluated using both the chaotic and conventional code generators.

A Matlab programs are developed to perform orbit determination process and to measure the bit energy to noise ratio required for the communication. The developed programs take into account the dynamic model of the satellite orbit including various orbital perturbations and simulate the path delay for ranging measurements including clock offsets, thermal, ionosphere and troposphere noise. Acceptable results were foreseen in comparison to the flight proven software tool.

\section{Keywords:}

Spread spectrum, orbit determination, dual ranging 


\section{Introduction:}

Spread Spectrum (SS) [1] - [5] has been defined as a means of transmission in which the signal occupies bandwidth much in excess of the minimum necessary to send the information. The band spread is accomplished by utilizing a "code" which is independent of the data and a synchronized reception with the code at the receiver is used for de-spreading and data recovery.

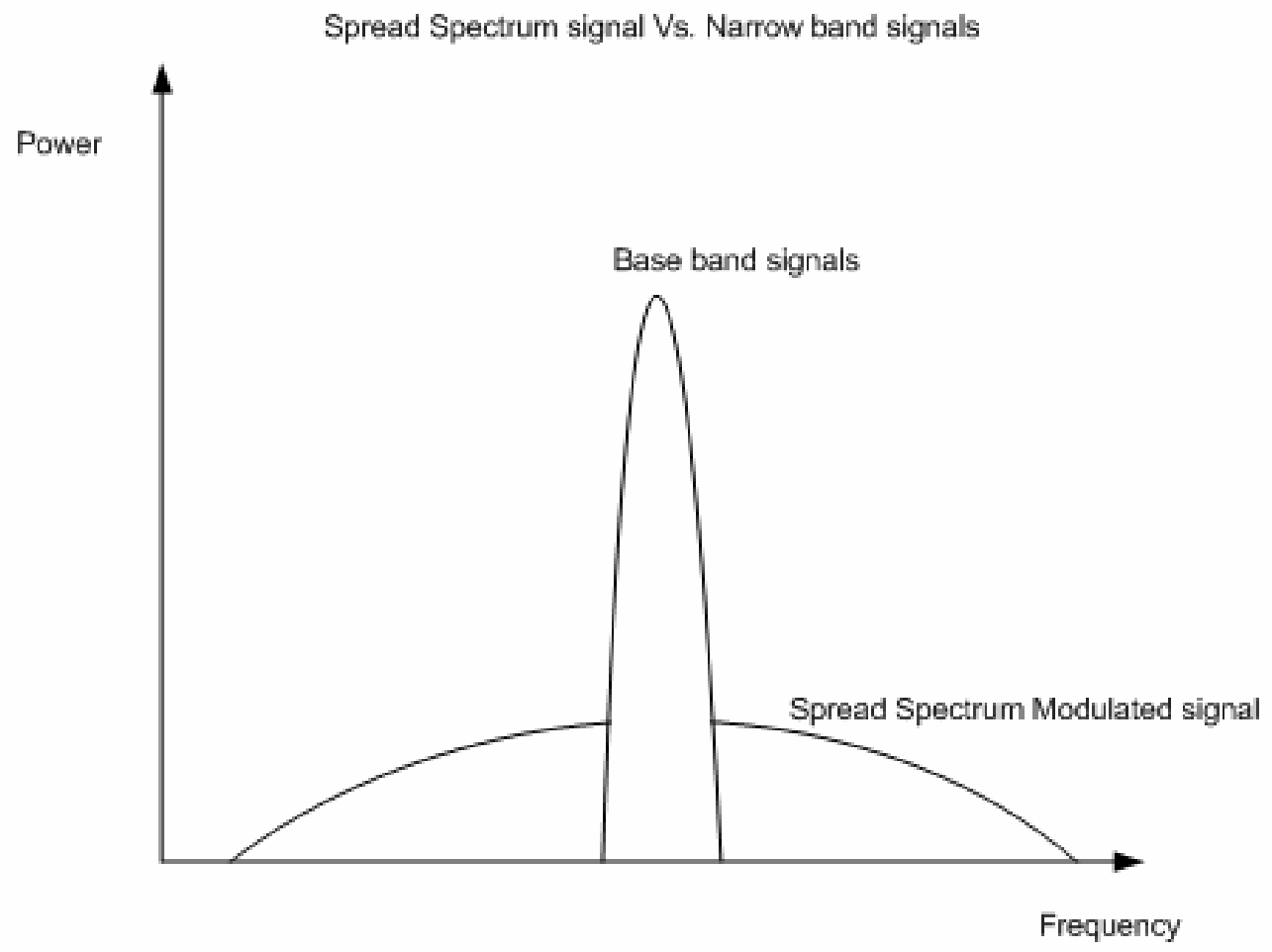

Figure (1): Base-band Signal / Spread Signal

The "code" [6] used for spreading the signal is a pseudo-random code that is mixed with the data to spread the signal in a statistically random matter. These codes are considered fast codes as they run the information bandwidth or data rate many times. These special "Spreading" codes are called "Pseudo Random" or "Pseudo Noise" codes. They are called "Pseudo" because they are not real white noise.

The cost paid is the need for a larger bandwidth which is already present due to the usage of the existing communication channels and the need for good synchronization at the receiver to detect the reception of the signal. 


\section{Direct sequence spread spectrum (DS/Ss) ranging technique:}

The used method is the direct-sequence spread spectrum ranging technique, as shown in figure (2). This technique has been used in GPS C/A (Clear/Acquisition) code and $\mathrm{P}$ (Precise) code.

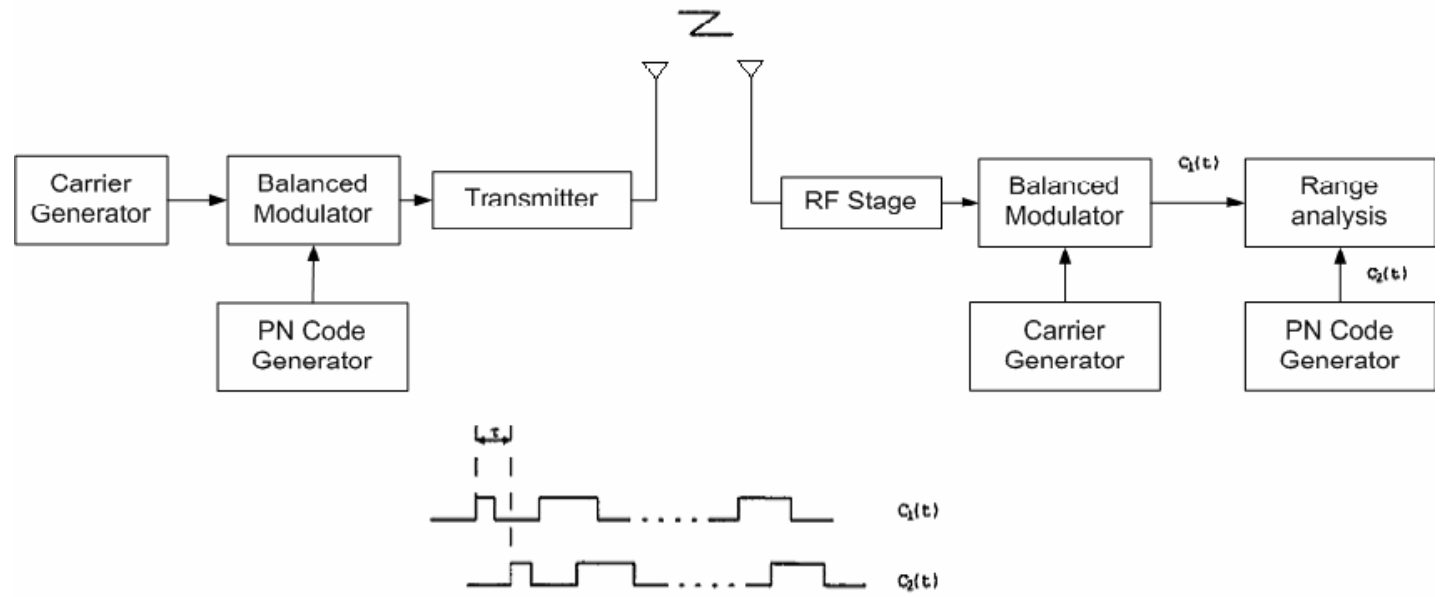

Figure (2): DS/Ss system

The difference between DS/Ss and CW ranging systems is that with DS/Ss the carrier is phase-shift modulated by a pseudo-random signal. At the receiver, the received signal is demodulated. By measuring the number of chips of the code delay between the signals being transmitted and received, one can determine uniquely the range from the transmitter to the receiver.

The spread spectrum technique has its advantage in that its phase is easily resolved, as shown in figure (3). By matching received PN code and the local PN code, a high time resolution can be achieved. If the repetition cycle of the PN code is long enough, the range between transmitter and the receiver can be determined without ambiguity.

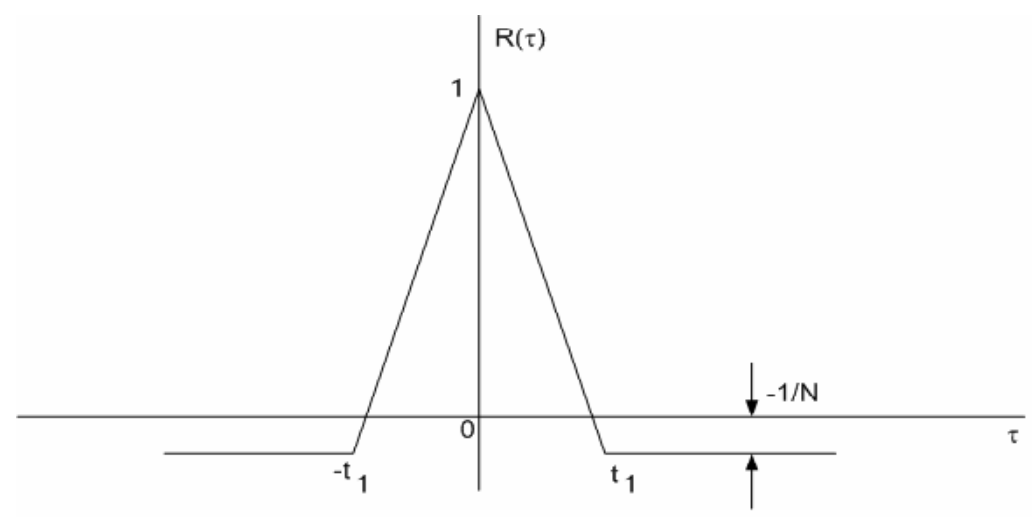

Figure (3): DS/Ss Correlation 
Proceedings of the $7^{\text {th }}$ ICEENG Conference, 25-27 May, 2010

\section{Advantages and disadvantages of DS/Ss ranging system}

\subsection{Advantages of DS/Ss ranging system:}

Spread spectrum ranging is also called 'Payload ranging' as it is transmitted via satellite payload channels. Compared to classical Pseudo-range systems, the use of payload signals has various advantages for the satellite operator [7].

The payload based ranging signal is always present; it doesn't need a dedicated activation, and has no impact on the satellite control activities while classical Pseudorange has to be actually activated by satellite commanding. Thereupon, tone ranging campaigns are limited in time, in contrary to payload methods, which provides continuous range.

Moreover, the accuracy of payload ranging is in general superior, mainly due to the large bandwidth and high signal-to-noise ratio. The latter two effects also make payload ranging an optimal solution for orbit determination.

\subsection{Disadvantages of DS/Ss ranging system}

The range resolution of a DS/Ss ranging system depends on the chosen chip rate and accuracy of synchronization of the local PN code with the received PN code. That is to say, to design DS/Ss ranging system with range resolution of $5 \mathrm{~cm}$, and assuming the accuracy of PN code synchronization of the transmitter and the receiver can discriminate to one-hundredth of a chip. The chip wavelength should be less than 5 meters $\left(=5 \times 10^{2} \times 100\right)$, and as the chip rate is equal to:

$$
\mathrm{R}_{\mathrm{c}}=c / \lambda \mathrm{c}
$$

The given $R_{c}=60 \mathrm{Mcps}$, is impractical for the applications with lower carrier frequencies. Increasing the resolution of a DS/Ss system requires increasing the chip rate or increasing synchronization accuracy of the system or both. Unfortunately, both methods are often limited to other factors in practical applications.

Another limitation with DS/Ss ranging systems is that only the phase resolvability of the modulating pseudorandom signals is used. The carrier phase information which can provide higher range resolution is discarded. 


\section{Additional block sets to the spread spectrum system:}

\subsection{Chaotic Code Generators:}

A chaotic dynamical system [8] is an unpredictable, deterministic and uncorrelated system that exhibits noise-like behavior through its sensitive dependence on its initial conditions which generates sequences similar to PN sequence. The chaotic dynamics have been successfully employed to various engineering applications such as automatic control, signals processing and watermarking.

Since the signals generated from chaotic dynamic systems are noise-like, super sensitive to initial conditions and have spread and flat spectrum in the frequency domain, it is advantageous to carry messages with this kind of signal that is wide-band and has high communication security. For this reason, numerous engineering applications of secure communication with chaos have been developed. The disadvantage of such system is the complexity to synchronize the receiver chaos sequence with local generated at the receiver end.

A direct application of chaos theory to telecommunication systems appears in a conventional digital spread spectrum where the information is spread over a wider band by using a chaotic signal instead of the usual periodic PN sequences.

Various non-linear dynamic systems are used in order to generate the chaotic sequence as: Tent map, logistic map. The state space description of the logistical map is:

$$
\mathrm{F}(\mathrm{x}, \mathrm{r})=\mathrm{r} \mathrm{x}(1-\mathrm{x})
$$

And the state space description of the tent map is:

$$
\mathrm{F}\left(\mathrm{x}_{\mathrm{n}}\right)=\mathrm{x}_{\mathrm{n}+1}=\mathrm{k}\left(1-\left|1-2 \mathrm{x}_{\mathrm{n}}\right|\right)
$$

One major difference between chaotic sequences and PN sequences is that the generated chaotic sequences are not binary. Therefore chaotic sequences must be transformed into binary sequences.

In order to transfer the real valued chaotic sequence $(x)$ to binary sequence, a threshold function $\theta_{\mathrm{t}}(x)$ is defined as,

$$
\theta_{\mathrm{t}}(w)= \begin{cases}0 & , x<t \\ 1 & , x \geq t\end{cases}
$$

The threshold value is chosen as an arithmetic mean of a large number of conservative values of $x$. Thus a binary sequence is obtained and is referred to as a chaotic threshold sequence. 


\subsection{Residue number system (RNS)}

Residue number system [9] has two inherent features that render the RNS attractive in comparison to binary representation. These two features are the carry-free arithmetic, and lack of ordered significance amongst the residue digits.

The first property implies that the operations related to the individual residue digits of different moduli are mutually independent because of the absence of carry information. The second property of the RNS arithmetic implies that some of the residue digits can be discarded without affecting the result, provided that a sufficiently "high dynamic range" is retained in the "reduced" system in order to unambiguously contain the result, as argument below.

From the first property, it is possible to selectively utilize RNS in Ss in order to decrease number of data transmitted and thus improving the system performance and to add security to the system through encrypting the data signal and converting arithmetic of large numbers to arithmetic of small numbers, thus improving the signal-to-noise ratio of the received signal and decreasing the bit error probability.

\section{System Model:}

The orbit determination using only ranging measurements - as shown in figure (4) approach is established to be independent from the angular measurements.

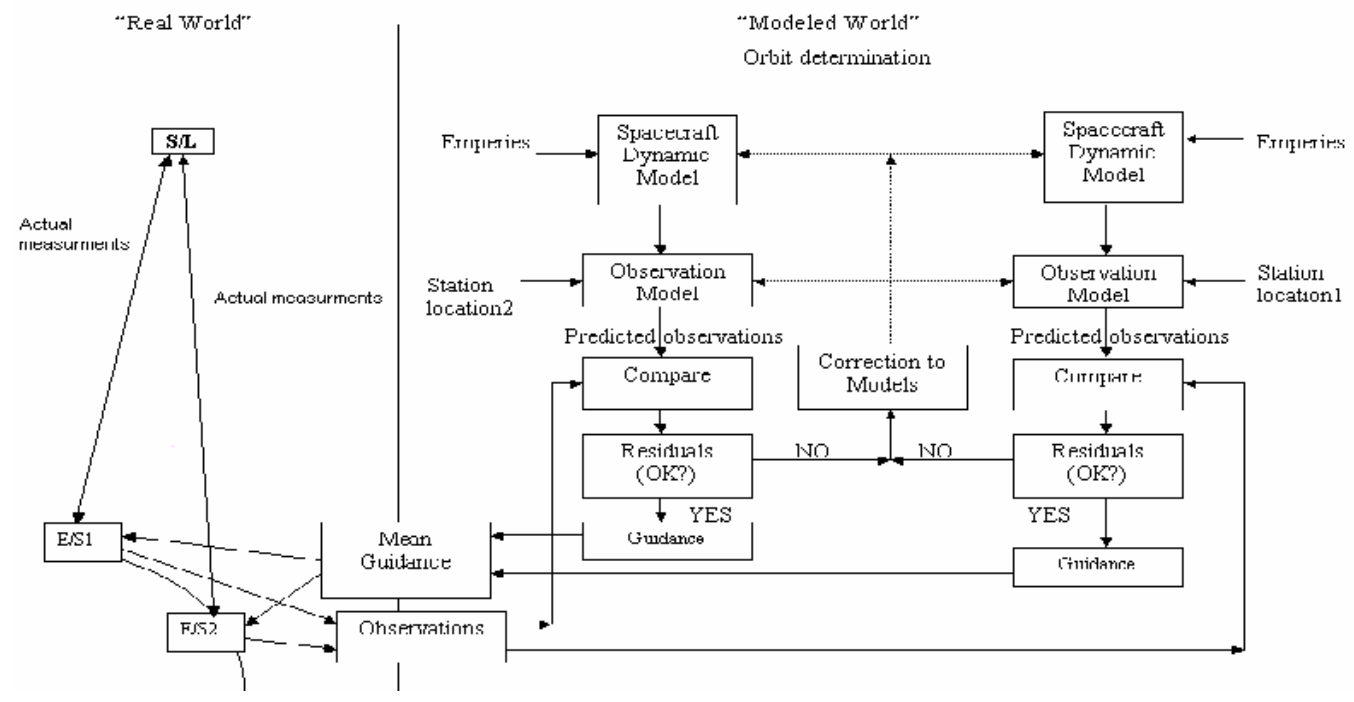

Figure (4): Dual ranging Algorithm

Where the orbit determination process is performed twice, one for each station, then the optimum orbit is generated from the mean error value of both orbits. 
In addition, to simulate the ranging measurements, figure (5) shows the system block using BPSK modulation/demodulation technique and adding to it binary to residue number system (RNS) conversion in order to improve the system performance.

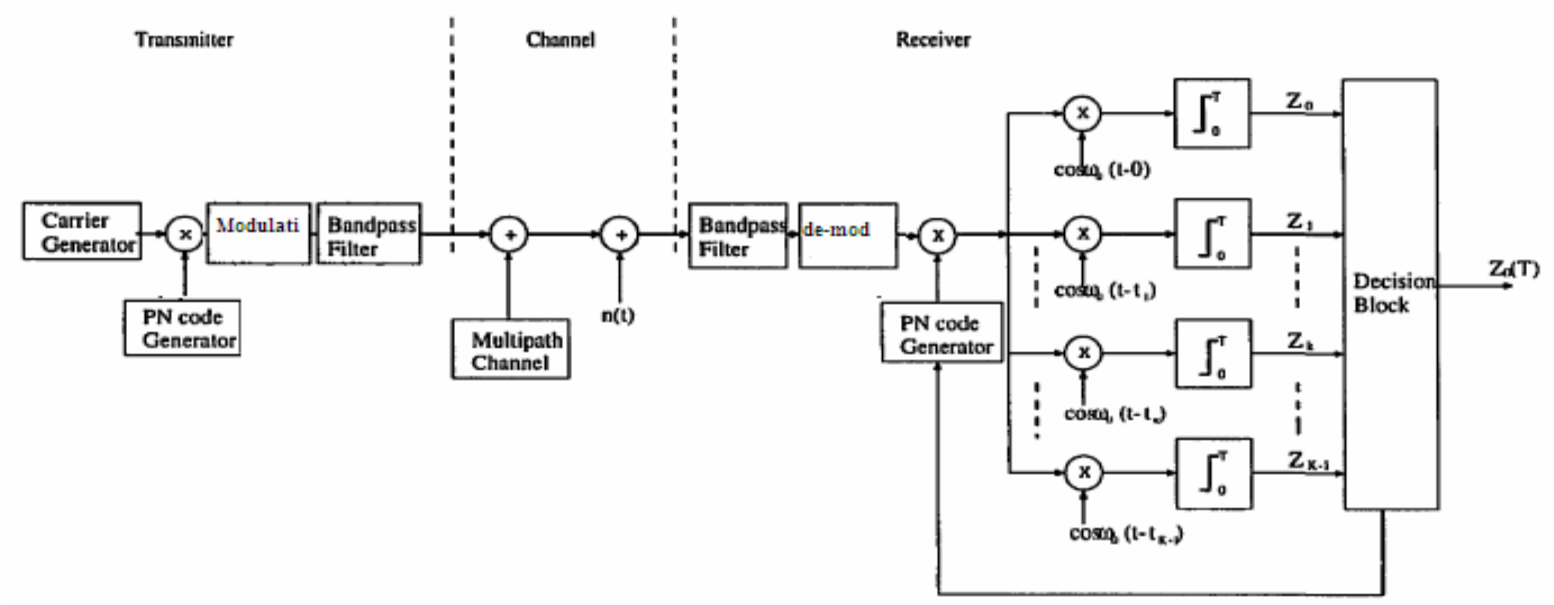

Figure (5): System Block diagram

The channel model uses a variable path delay $\left(\mathrm{t}_{\mathrm{k}}\right)$ that simulates the distance between the earth station and the satellite. The channel noise is assumed to be additive, white, zero-mean Gaussian process, with two-sided noise power spectral density of $N_{o} / 2$ at the receiver input.

At the receiver, the demodulated signal is sent to a bank of correlators to find the signal delay and the carrier phase relative to the local carrier and the PN code. The output of the $\mathrm{k}^{\text {th }}$ correlator can be represented as:

$$
\mathrm{Z}_{\mathrm{k}}\left(\mathrm{T}, \varepsilon, \mathrm{t}_{\mathrm{k}}\right)={ }_{0} \int^{\mathrm{T}} \mathrm{s}(\mathrm{t}) \mathrm{b}(\mathrm{t}-\varepsilon) \sqrt{2} \cos \left[\omega_{0}\left(\mathrm{t}-\mathrm{t}_{\mathrm{k}}\right)+\theta\right] \mathrm{dt}+\mathrm{n}_{\mathrm{k}}
$$

A decision block is needed in order to detect and count up the number of delayed chips. The auto-searching process for the range measurement of the receiver is as follows:

Step 1: De-spread the received signal and do the correlation operation.

Step 2: Choose the largest output from $Z_{k}, k E\{\mathrm{O}, \ldots K-1\}$, compare $Z_{k}$ with the threshold $\mathrm{A}_{0}$

Step 3: If Max $Z_{k}<\mathrm{A}_{0}$, shift $b(t)$, counter +1 , repeat Step 1, 2; else, $Z_{\mathrm{k}}=\operatorname{Max} Z_{k}$

The value of the counter and $k_{o}$ can determine the time delay of the received signal through the following equation:

Time delay $=$ counter $\times \Delta \mathrm{T}+\left(\mathrm{k}_{0} / \mathrm{K}\right) \mathrm{T}_{0}$

To avoid an ambiguous decision, $\Delta \mathrm{T}$ should be less than or equal to $\boldsymbol{T}_{\boldsymbol{o}}$. The threshold $\mathbf{A}_{\mathbf{0}}$ should be chosen so that the PN signal lines-up within $\boldsymbol{T}_{\boldsymbol{o}}$ can be detected. 


\section{Simulation:}

Various simulations were performed in order to study the performance of spread spectrum ranging over AWGN channel in comparison to tone ranging, and finally compute the orbit using spread spectrum ranging.

\subsection{The bit error probability for various code generators}

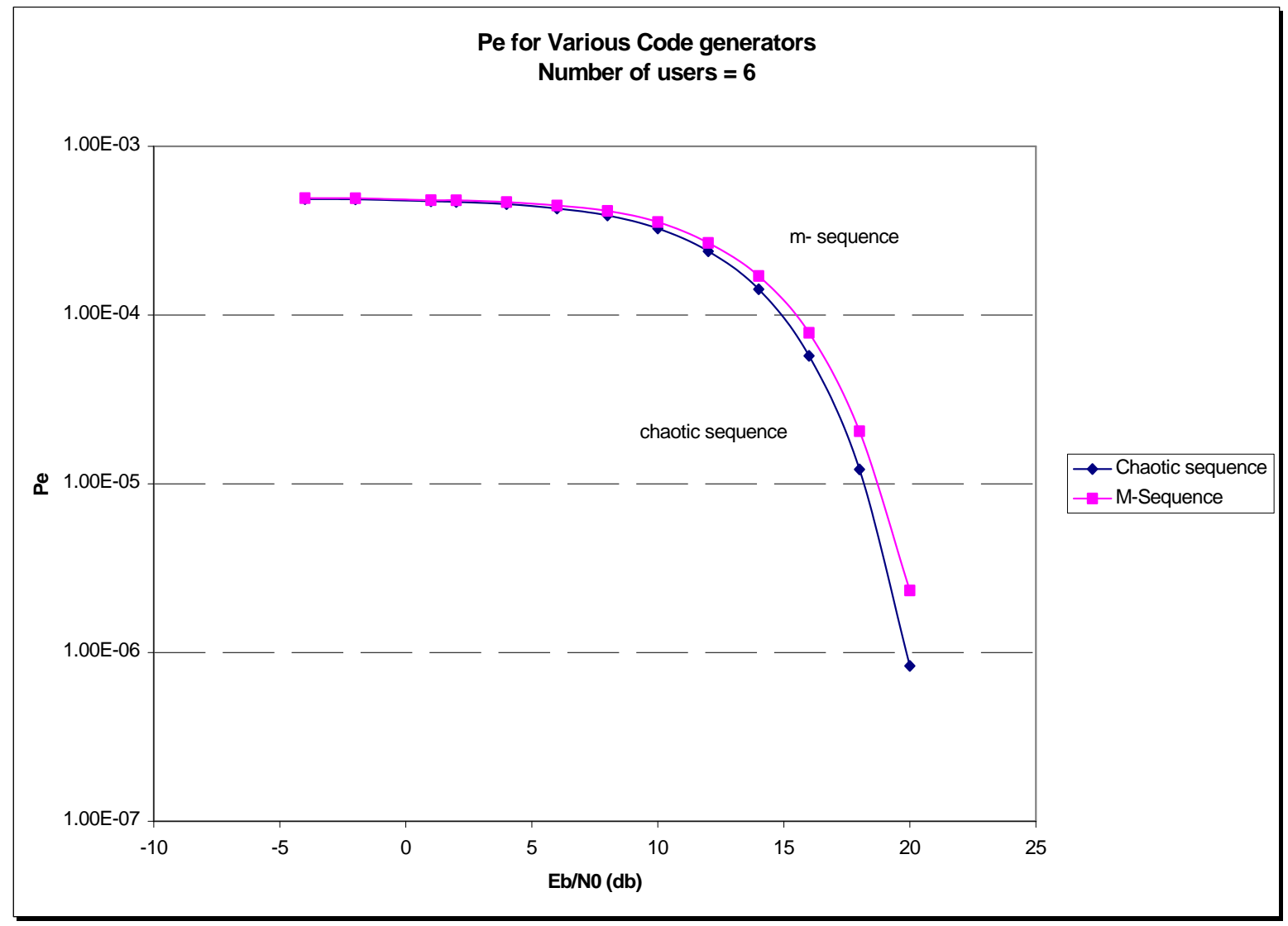

Figure (6): Bit error probability $\left(\mathrm{P}_{\mathrm{e}}\right)$ for 6 - users DS-SS system using: $\mathrm{m}$ - sequence / Logistic map codes

The bit error probability $\left(\mathrm{P}_{\mathrm{e}}\right)$ for Chaos-based spreading sequence is improved by around $15 \%$ at $\mathrm{E}_{\mathrm{b}} / \mathrm{N}_{\mathrm{o}}=15 \mathrm{db}$, thus at any fixed BER their $>15 \%$ more users can be allocated for free chaotic-based codes.

Thus, although m-sequence may be good for single station ranging but it is not suitable for multiple access applications. 


\subsection{Effect of adding RNS to Ss system}

The effect of selected RNS (moduli 57 9) to the system is studied by measuring the $\mathrm{P}_{\mathrm{e}}$ for the communication system versus signal to noise ratio, as seen in figure (7).



Figure (7): Pe Vs $\mathrm{E}_{\mathrm{b}} / \mathrm{N}_{0}$ for BPSK Ss System

Figure (7), shows that when a selected RNS (moduli 57 9) is used in the SS system, the signal to noise ratio (SNR) is improved due to the increase in the code rate, thus decreasing the bit error probability. The performance improvement of approximately $2 \mathrm{~dB}$ at a bit error probability of $10^{-5}$ is foreseen.

\subsection{The optimum $E_{b} / N_{0}$ required for communication}

Using tone ranging and spread spectrum ranging system, the simulation results provide:

- An optimum $\mathrm{E}_{\mathrm{b}} / \mathrm{N}_{0}$ foreseen using Tone ranging is $\mathbf{1 4} \mathbf{~ d b}$.

- An optimum $\mathrm{E}_{\mathrm{b}} / \mathrm{N}_{0}$ foreseen using Chaotic-Sequence is $\mathbf{9} \mathbf{d b}$.

This is due to the following reasons:

- Only one earth station is used as tone ranging cannot use multiple stations at the same time.

- Tone ranging need higher power due to the need to transmit multiple tones sequentially for ambiguity resolution, leading to an increase in the total transmitted sequence and thus degrades the performance as it requires more channel bandwidth due to the increased size of the transmitted data. 


\subsection{Orbit determination computation using dual ranging spread spectrum} Inputs:

- Number of Ground stations: 2

- Number of Data bits (used as a station identification bits) $=5$

- Initial value of the chaotic sequence $=-0.45$

Noting that due to the fact that spread spectrum ranging measurements are simulated not real measurements - the resulted orbital parameters using these values are not totally concede with the flight proven parameters.

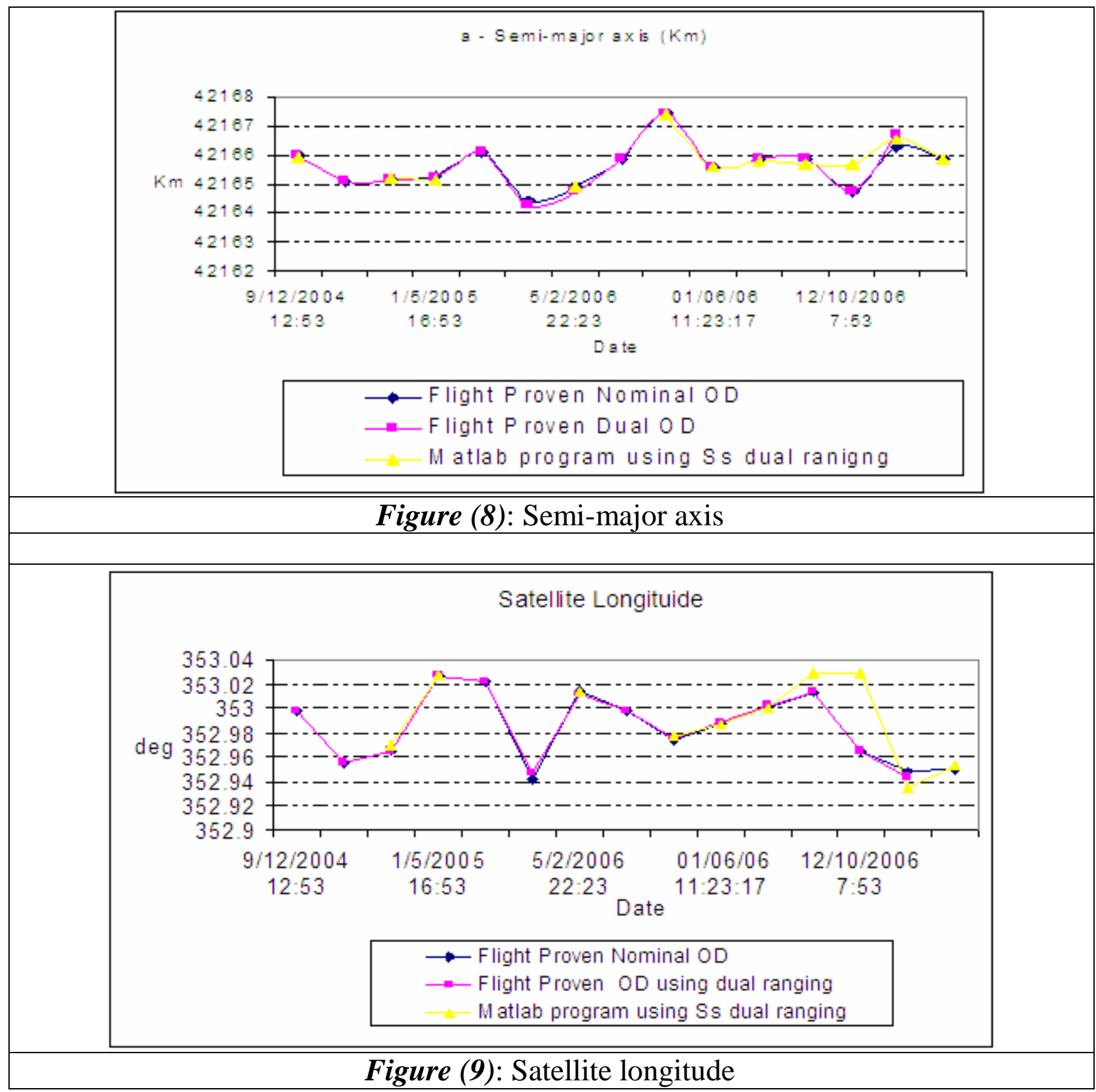




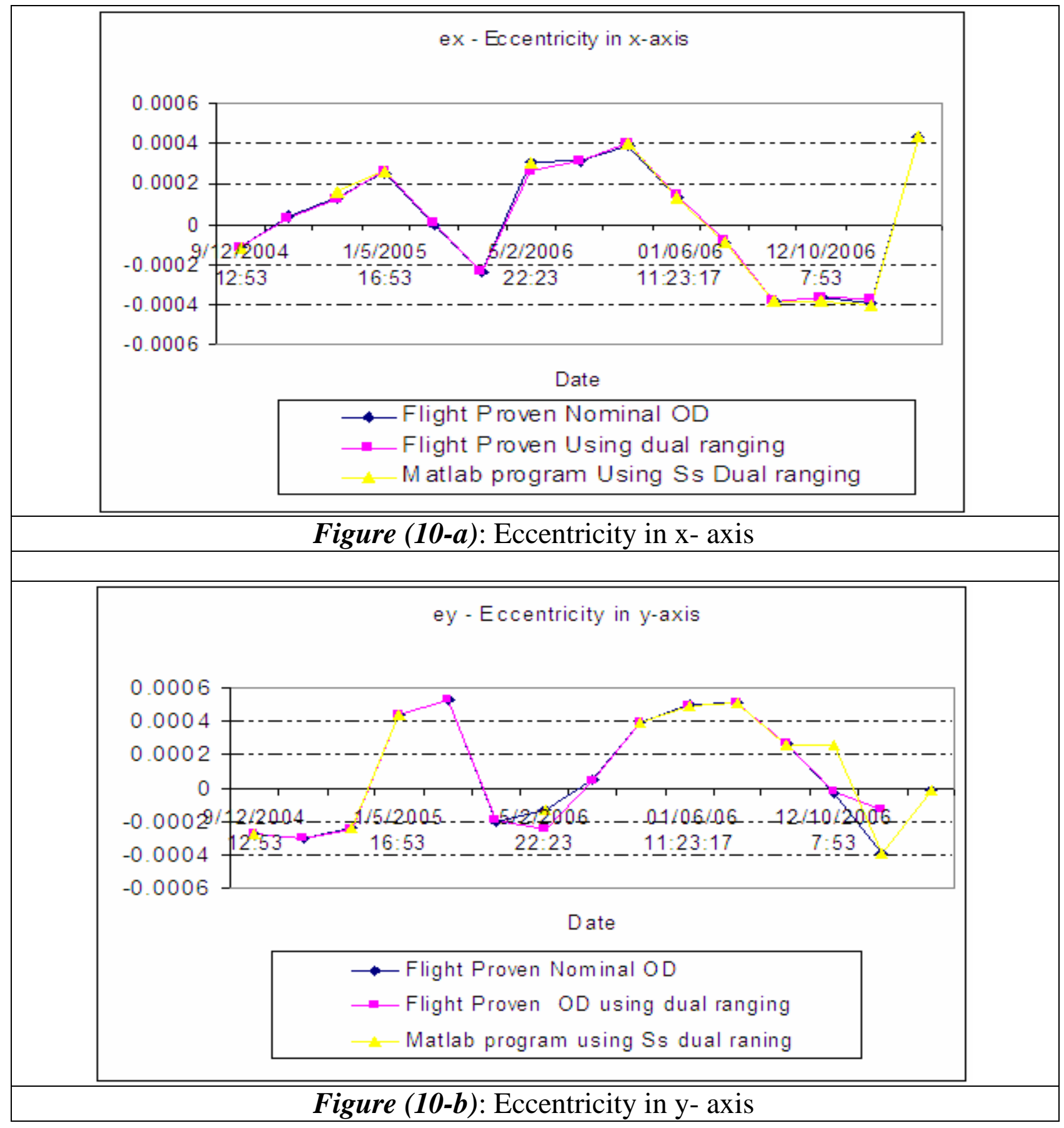






The above orbital parameters are computed taking into account the predicted orbital perturbations as the path delay simulations (clock offsets of the satellite and the ground station, thermal, Ionosphere and troposphere noise, the orbital perturbations), and the channel delay that simulate the round trip delay between the earth station and the satellite. Thus, these measurements provide realistic ranging values.

These orbital parameters are compared to the orbital parameters that are generated from a flight proven software tool using nominal measurements and also with only ranging measurements. Henceforth, acceptable results were foreseen. 


\section{Conclusions:}

This paper demonstrates that the spread spectrum communication is optimized through the usage of suitable residue number system to convert with it the Tx data stream. Thus, improving the signal-to-noise ratio due to the increase in the code rate, and thus decreasing the bit error probability.

Utilization of chaotic sequence generators instead of conventional PN code generators for spreading the data sequence. This provides better security and reduced multiple access interference (MAI) in comparison to m-sequence code generator.

Validation of the orbit generated using simulated spread spectrum ranging and comparing it to the orbit determination generated from actual tone ranging measurements. The results showed that tone ranging need higher power due to the need to transmit multiple tones sequentially for ambiguity resolution, leading to an increase in the total transmitted sequence and thus degrade the performance as it need more channel bandwidth due to the increase size of the transmitted data.

\section{References:}

[1] N.B chakrabarti , A. K . Datta, "introduction to the principles of digital communication", New Age Publishers, 2007.

[2] Erik Storm, Tony Ottosson, Arne Svensson, "An introduction to spread spectrum systems", Department of signals and systems, Chalmers university of technology, Sweden, 2002.

[3] Raymond L. PICKHOLTZ, "Theory of spread spectrum communication - A tutorial", IEEE Trans. Communication, vol. 30, No.5, May 1982.

[4] Ryuji Kohno, Reuven Meidan, and Laurence B. Milstein, "Spread Spectrum Access Methods for Wireless Communications", IEEE Communication magazine, January 1995.

[5] Yong Luo, "Spread Spectrum Ranging System - Analysis and Simulation", Master Thesis in Electronic systems engineering - University of Regina, Saskatchewan, March 1998.

[6] Ipsita Bhanja, "Performance comparison of various spreading codes in spread spectrum modulation in ranging technique", Proc of national conference on range technology, pp30-35, 2006

[7] G. Harles, J. Wouters, B. Fritzsche , F. Haiduk, "Operational Aspects of an Innovative, DVB-S based, Satellite Ranging Tool" - SpaceOps Conference , 2004. 
[8] S. Mandal and S. Banerjee, "A chaos-based spread spectrum communication system" Nat. Conf. Nonlinear Sys. Dynamics, Indian Institute of Technology, Kharagpur, Dec 28-30, 2003.

[9] Lie-Liang, Lajos Hanzo, "Performance of residue number system based DSCDMA over multi-path fading channels using orthogonal sequences", university of Southampton, UK, July 1999.

\section{Nomenclatures:}

Ds... Direct Sequence

C/A Code... Clear/Acquisition code

P Code... Precise or Protected code

N... Length of PN sequence

$t_{1} \ldots$ Chip duration

CW... Continuous Wave

PN... Pseudo noise generator

RF... Radio frequency

Ss... Spread Spectrum

c... speed of light

$\lambda_{\mathrm{c}} \ldots$ wavelength of a chip

$\mathrm{R}_{\mathrm{c}} \ldots$ chip rate

$\mathrm{n}_{\mathrm{k}} \ldots$ Noise component

b... PN signal

$\theta \ldots$ Carrier synchronization error

$\varepsilon \ldots$ local PN code shift

T... duration of measurement time

$\Delta \mathrm{T}$... Step length

$\mathrm{T}_{0} \ldots$ period of the carrier

$t_{k} \ldots$ Local carrier shift of the $k^{\text {th }}$ correlator

t... threshold value

$\mathrm{s}(\mathrm{t}) \ldots$ received signal

r... bifurcation parameter

F... transformation mapping function,

k... Arbitrary constant selected by the designer to make a chaotic system.

SNR... Signal to Noise Ratio

Tx... Transmitted 